Fumiaki Ayabe · Motonobu Katoh · Toshiaki Inoue

Natalay Kouprina · Vladimir Larionov · Mitsuo Oshimura

\title{
A novel expression system for genomic DNA loci using a human artificial chromosome vector with transformation-associated recombination cloning
}

Received: 15 July 2005/ Accepted: 11 August 2005/Published online: 18 October 2005

(C) The Japan Society of Human Genetics and Springer-Verlag 2005

\begin{abstract}
Following the recent completion of the human genome sequence, genomics research has shifted its focus to understanding gene complexity, expression, and regulation. However, in order to investigate such issues, there is a need to develop a practical system for genomic DNA expression. Transformation-associated recombination (TAR) cloning has proven to be a convenient tool for selective isolation of a genetic locus from a complex genome as a circular YAC using recombination in yeast. The human artificial chromosome (HAC) vector containing an acceptor loxP site has served as a platform for the reproducible expression of transgenes. In this study, we describe a system that efficiently expresses a genetic locus in mammalian cells by retrofitting a TAR-YAC with the donor loxP site and loading it onto the HAC vector by the Cre/loxP system. In order to demonstrate functional expression of genomic loci, the entire human hypoxanthine phosphoribosyl transferase (HPRT) locus contained in a $100 \mathrm{~kb}$ YAC was loaded onto the HAC vector and was shown to complement the genetic defect in Hprt-deficient $\mathrm{CHO}$ cells. Thus, the combination of TAR cloning and the HAC vector may serve as a powerful tool for functional genomic studies.
\end{abstract}

\footnotetext{
F. Ayabe $\cdot$ M. Oshimura $(\bowtie)$

Department of Biomedical Science,

Institute of Regenerative Medicine and Biofunction, Graduate School of Medical Science, Tottori University, 86 Nishi-cho, Yonago, Tottori 683-8503, Japan

E-mail: oshimura@grape.med.tottori-u.ac.jp

Fax: + 81-859-348134

M. Katoh $\cdot$ T. Inoue $\cdot$ M. Oshimura

Department of Human Genome Science,

Graduate School of Medical Science, Tottori University,

86 Nishi-cho, Yonago, Tottori 683-8503, Japan

N. Kouprina $\cdot$ V. Larionov

Laboratory of Biosystems and Cancer,

National Cancer Institute, NIH,

Bldg. 37, Room 5032, 90000 Rockville Pike,

Bethesda, MD 20892, USA
}

Keywords Genomic expression · Human artificial chromosome (HAC) vector · Transformation-associated recombination (TAR) cloning $\cdot$ TAR-HAC combination system $\cdot H P R T$ (hypoxanthine guanine phosphoribosyl transferase)

\section{Introduction}

Spaciotemporal regulation of gene expression in cells is achieved by complex mechanisms such as alternative splicing (Faustino and Cooper 2003; Graveley 2001; Wen et al 2004), alternative promoter-enhancer usage (Landry et al 2003), intronic gene expression (Conrad et al 2002), and expression of inhibiting RNAs (Tufarelli et al 2003). DNA polymorphisms affect these regulatory systems, and the composition of these polymorphisms produces a haplotype which represents the phenotype of cells in an individual (Hoehe et al 2003). Most of the conventional gene expression studies employ cDNA or minigene constructs. However, they cannot easily recapitulate the context of a genomic locus (Garrick et al 1998; Whitelaw et al 2001). The delivery of a gene as a genomic locus would enable control of gene expression by a native promoter and regulation of endogenous enhancer and silencer elements.

A new approach has recently emerged that allows entire genes and large chromosomal regions to be selectively and accurately isolated from complex genomes (Kouprina et al 1998). This system, called TAR cloning, employs in vivo recombination in yeast in order to clone genomic DNA. Yeast spheroplasts are transformed with genomic DNA along with a linearized TAR cloning vector, which contains yeast centromere, auxotrophic selection marker, and unique $5^{\prime}$ and $3^{\prime}$ sequences specific to the gene of interest. Linearization of the vector bears the recombinogenic target sequence at both ends. Transformation-associated recombination between the gene-specific sequences in the vector and the gene-containing genomic fragment 
leads to the establishment of a circular YAC. Propagation of the YAC in the yeast cell depends on the acquisition of autonomously replicating sequences in the cloned genomic segment. This allows an entire gene to be isolated without interruption by restriction digestion, which is inevitable in conventional in vitro cloning methods (Kouprina and Larionov 2003). Since TAR cloning eliminates laborious steps such as constructing a new genomic library and screening to obtain the desired clones, it is applicable for isolating haplotype alleles from an individual (Kim et al 2003; Leem et al 2002).

Gene delivery into cells for functional expression is sometimes hampered by problems such as irreproducible expression levels among transfectants. This is thought to result from the highly variable number of copies integrated into the host genome and from position effects on gene expression due to random integration (Garrick et al 1998; Whitelaw et al 2001). To overcome these problems, we have established a HAC vector system (Katoh et al 2004). The HAC vector, produced by truncating the distal parts of both p- and q-arms from human chromosome 21 using chromosome engineering, is equipped with an acceptor loxP site at which circular DNA can be loaded by the $\operatorname{Cre} / \operatorname{lox} P$ system. In this study, we tested the loading of a genomic clone isolated by the TAR method into a HAC vector and verified the functional expression of a gene from the genomic locus introduced, utilizing the well characterized human HPRT gene as a model.

\section{Materials and methods}

Cell culture and establishment of

Hprt-deficient $\mathrm{CHO}$ cells

$\mathrm{CHO}$ cells carrying the $21 \Delta \mathrm{pqHAC}$ vector were maintained in Ham's F-12 nutrient mixture (Invitrogen, Carlsbad, CA, USA) with 10\% fetal bovine serum (FBS: JRH Biosciences, Lenexa, KS, USA) and $8 \mu \mathrm{g} / \mathrm{ml}$ blasticidin S hydrochloride (Funakoshi, Tokyo, Japan). Hprt-deficient $\mathrm{CHO}$ cells were established by culturing the CHOHyg8 clone (Katoh et al 2004) in Ham's F-12 medium supplemented with $10 \%$ FBS, $8 \mu \mathrm{g} / \mathrm{ml}$ blasticidin $\mathrm{S}$ hydrochloride, and $15 \mu \mathrm{M}$ 6-thioguanine (Kanto Chemicals, Tokyo, Japan).

\section{Construction of the retrofitting vector}

The retrofitting vector $\mathrm{BRV} 1 / \mathrm{hCMV}$-loxP was constructed as follows: a $0.8-\mathrm{kb}$ hCMV-loxP fragment was obtained by digesting pBS226 (Invitrogen) (Fukushige and Sauer 1992) with SpeI and NotI, and then cloned into AvrII/NotI sites in the BRV1 vector (Kouprina et al 1998).
Retrofitting of the YAC and conversion to BAC

A standard lithium acetate transformation procedure (Ito et al 1983) was used to retrofit the circular HPRTYAC isolated by TAR cloning (Kouprina et al 1998). Briefly, yeast containing the HPRT-YAC was treated with lithium acetate, then the linearized retrofitting vector was introduced, and it was plated on SD-URA plates. Ura + His + clones were isolated and propagated. The yeast chromosome-size DNA was prepared in agarose plugs, treated with agarase, and then electroporated with the linearized retrofitting vector into DH10B competent cells (Invitrogen) using Gene Pulser (BioRad, Hercules, CA, USA). Transformants were selected on LB plates with chloramphenicol.

\section{Pulsed field gel electrophoresis}

Agarose plugs prepared from yeast transformants containing circular YACs were exposed to X-rays (100 Gy) for $30 \mathrm{~min}$ (Hitachi Medico, Tokyo, Japan). Chromosome-size DNA was separated using CHEF DR-II pulsed field gel electrophoresis apparatus (Bio-Rad), on $1 \%$ agarose gel in $0.5 \times \mathrm{TBE}$ buffer at $14{ }^{\circ} \mathrm{C}$. The runtime was $24 \mathrm{~h}$ at $6 \mathrm{~V} / \mathrm{cm}$ with a $60-120 \mathrm{sec}$ switch time ramp.

\section{Southern blotting}

After separation by agarose gel electrophoresis, DNA was transferred to Hybond $\mathrm{N}+$ membranes (Amersham, Piscataway, NJ, USA) in $0.4 \mathrm{~N} \mathrm{NaOH}$ solution. The membranes were hybridized overnight at $65^{\circ} \mathrm{C}$ with ${ }^{32} \mathrm{P}$-labeled probes and washed twice at $65^{\circ} \mathrm{C}$ in $0.1 \times$ SSC and $0.1 \%$ SDS for 15 min. Radioactivity on the membrane was detected and visualized by an image analyzer, BAS2000 (Fuji Photo Film, Tokyo, Japan).

\section{Restriction analysis of $H P R T-\mathrm{BAC} / \mathrm{YACs}$}

The endonuclease restriction analysis was performed as follows. BACs were isolated using the standard method (Qiagen, Tokyo, Japan). Ten micrograms of BAC DNA were incubated overnight with $4 \mathrm{U}$ of EcoRI per $1 \mu \mathrm{g}$ DNA in $1 \times$ buffer (Wako, Osaka, Japan) at $37^{\circ} \mathrm{C}$. Digested fragments were separated by electrophoresis on a $0.8 \%$ agarose gel in $0.5 \times \mathrm{TBE}$.

Transfection of $H P R T-\mathrm{BAC} / \mathrm{YACs}$ into $\mathrm{CHOHyg} 8 \Delta H$ prt cells

$H P R T$-loxP/BAC/YACs were isolated using a standard alkaline lysis procedure, purified on Qiagen columns, and transfected into Hprt-deficient CHOHyg8 $\mathrm{CHprt}$. A total of $4 \times 10^{5} \mathrm{CHOHyg} 8 \Delta H$ prt cells were transfected 
with $5 \mu \mathrm{g}$ of the HPRT-loxP/BAC/YACs and $1 \mu \mathrm{g}$ of the Cre expression vector pBS185 (Invitrogen) by lipofection with $10 \mu \mathrm{l}$ of Lipofectamine 2000 reagent (Invitrogen), following manufacturer's instructions. After being cultured for $24 \mathrm{~h}$ in basic growth medium, cells were then cultured in a medium containing $800 \mu \mathrm{g} / \mathrm{ml}$ G418 (Invitrogen). Fourteen days later, drug-resistant colonies were isolated and expanded for further analysis.

\section{PCR analysis of genomic DNA}

DNA from cell lines was prepared with Puregene solution (Gentra Systems, Minneapolis, MN, USA). Four pairs of primers were used for PCR:

- IN1L (5'-CCCCATCAGCCTCTGGTATCTTAGC$\left.3^{\prime}\right)$ and IN1R (5'-AGCCAGCACCTCAGATATACA-3'), specific for the intron 1 sequence (516-bp)

- 46L (5'-TGCTGGGATTACACGTGTGAACC-3') and 47R (5'-GACT CTGG CTAGAGTT CCTTC TTCC-3'), specific for exon 2 (575-bp)

- exon9F (5'-ATTAAACTAATGTGATAGACTAC T GCTTTG-3') and exon9R (5'-TTCAATGTTTCACT CAATAGTGCTGTGG-3'), specific for exon 9 (530bp) of the HPRT gene, and CMVneo586 (5'-CGT AACAACTCCGCCCCATT-3') and CMVneo972 (5'-CGGACAGGTCGGTCTTGACA-3') for junction with $\mathrm{Neo}$ reconstruction (387-bp).

\section{RNA expression analysis of $H P R T$}

Cytoplasmic RNA was extracted from $5 \times 10^{6}$ cells using the NucleoSpin RNAII kit (Macherey-Nagel, Düren, Germany), following the manufacturer's protocol. $\mathrm{Hu}-$ man HPRT RNA was detected by RT-PCR using the primers

- HPRT5'L(5'-TCCTCCTCCTGAGCAGTCA-3') and - HPRTexon3R (5'-CATCTCGA GCAAGACGT TC A-3').

Fluorescence in situ hybridization analysis

Metaphase chromosomes were prepared from the exponentially growing cells in cultures, and fluorescence in situ hybridization (FISH) was carried out by standard methods (Kugoh et al 1999). The probe used was digoxigenin (Roche, Basel, Switzerland)-labeled human Cot-1 DNA (Invitrogen). The digoxigenin signal was detected by an anti-digoxigenin-rhodamine complex (Roche). The chromosomes were counterstained with DAPI (Sigma, St. Louis, MO, USA). Images were captured using a microscope (Nikon, Japan) equipped with a photometric CCD camera, digitally processed, and visualized with the Argus system (Hamamatsu Photonics, Hamamatsu, Japan).

\section{Results}

Retrofitting of the HPRT-YAC with loxP

The HPRT1 gene is located on human chromosome Xq26.2 and encodes a purine salvage enzyme. Mutations in this gene result in Lesch-Nyhan syndrome (MIM 300322), a neurodevelopmental disorder characterized by self-injurious and abnormal motor behavior (Caskey and Kruh 1979). In our previous study, the HPRT1 locus was selectively isolated as a circular YAC from a normal human fibroblast cell line MRC5 using the TAR cloning method (Kouprina et al 1998). A 381-bp genomic DNA sequence approximately $12 \mathrm{~kb}$ downstream of the last exon (exon 9) of the HPRT gene was used as a specific hook for recombination at the $3^{\prime}$ end of the locus. An 189-bp Alu-consensus sequence was used as the other hook for recombination at an upstream region of the locus. The size of the isolated circular YAC was $\sim 100 \mathrm{~kb}$, suggesting that the $40 \mathrm{~kb}$ sequence upstream of exon 1 was included in the HPRT-YAC. We first constructed the retrofitting vector $\mathrm{BRV} 1-\mathrm{hCMV} /$ lox $P$ by modifying a $\mathrm{BAC}$ vector BRV1 using homologous recombination in yeast (Kouprina et al 1998) in order to equip the HPRT-YAC with a hCMV promoter and loxP site (Fig. 1a, b). A selectable marker URA3 was introduced into BRV1-hCMV/loxP for selection in yeast after transformation (Fig. 1a). BRV1-hCMV/loxP was linearized with BamHI, leaving free ends homologous to the circular YAC vector, and used for transformation of yeast VL6-48 containing HPRT-YAC, 13-2. Transformants were selected on SD-URA plates and replica plated on SD-HIS and SD +5FOA plates. Successful transformants grew on SD-HIS but not on $\mathrm{SD}+5 \mathrm{FOA}$

Transformation of 13-2 yielded 17 colonies that grew on SD-URA and SD-HIS but not on SD + 5FOA. Retention of intron 1, exon 2, and exon 9 of the HPRT gene was confirmed by PCR analysis (data not shown). The HPRT-YACs were linearized by exposure to $\mathrm{X}$ rays and separated by pulsed field gel electrophoresis, followed by Southern blotting with a human Cot-1 probe (Fig. 2b). Replacement of the ColE1 replication origin and Ampicillin resistance gene with the retrofitting vector was expected to increase the YAC size (Fig. 1a). Slightly shifted bands were detected in the retrofitted YACs, compared to the original HPRTYAC 13-2, with one exception (clone\#45). High molecular weight (HMW) DNA from the transformants was digested with EcoRI and subjected to Southern blotting with the HPRT cDNA probe (Fig. 2b). The DNA sequence information predicted that the nine exons are dispersed in four EcoRI fragments (Fig. 2a). Southern blotting showed the expected fingerprinting pattern of restriction fragments in 15 out of 17 transformants. Restriction fragments detected in the retrofitted YACs were identical to those in the 
a

a Retrofitting Vector

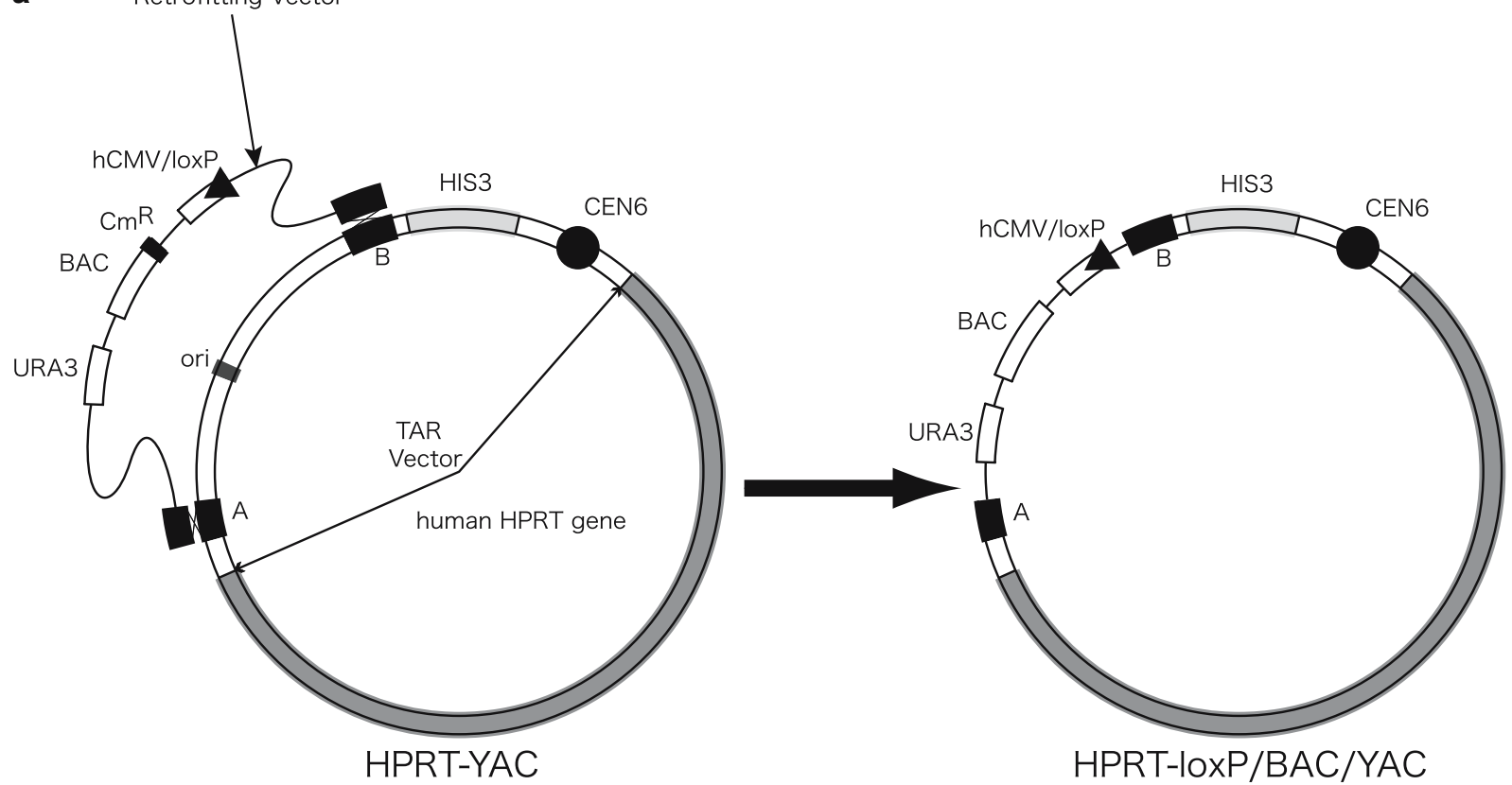

b

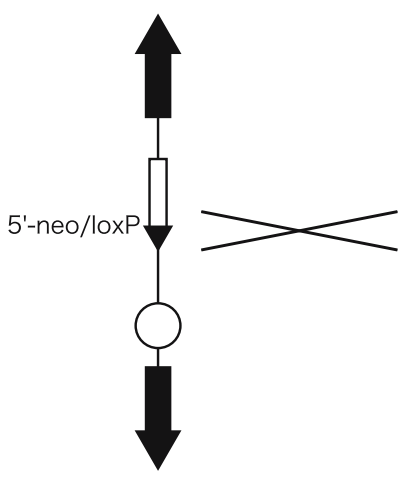

$21 \triangle \mathrm{pqHAC}$ vector

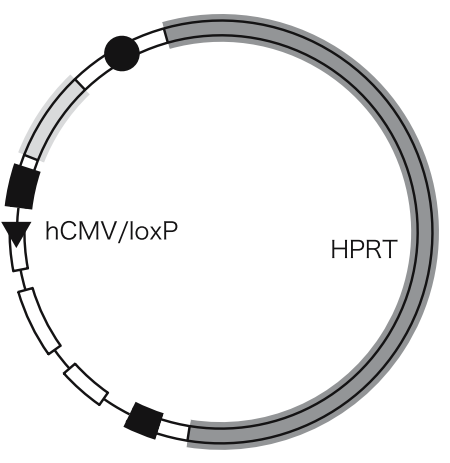

HPRT-IoxP/BAC/YAC
Cre recombinase expression vector
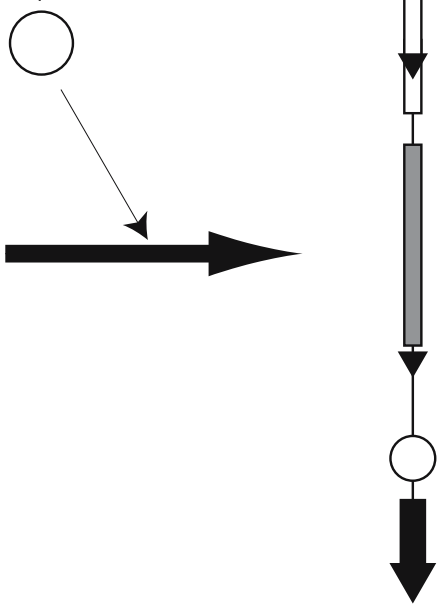

HPRT
Fig. 1a,b Diagram of HPRT-YAC retrofitting and loading to the $21 \Delta$ pqHAC vector. A circular YAC carrying the human HPRT locus was first retrofitted by recombination with the retrofitting vector derived from BRV1. It contains the F-factor (BAC) origin of replication, the chloramphenicol acetyltransferase $\left(\mathrm{Cm}^{\mathrm{R}}\right)$ gene, the URA3 yeast-selectable marker, two targeting sequences, A and B,

parental YAC, suggesting that the HPRT locus in the YACs maintained genomic integrity. Arbitrarily chosen $H P R T$-hCMV/loxP YAC/BAC was transferred into $E$. coli cells by electroporation for further BAC isolation. Retention of intron 1, exon 2, and exon 9 of the HPRT gene in isolated BACs was confirmed by PCR analysis (data not shown). and the hCMV-loxP sequence (a). The retrofitted YAC converted to $\mathrm{BAC}$ was isolated and transfected into Hprt-deficient $\mathrm{CHO}$ cells along with the Cre recombinase expression vector. Site-specific insertion was verified by reconstructing the functional $N e o^{R}$ gene, which confers resistance to G418 (b)

Isolation of Hprt-deficient derivatives from $\mathrm{CHO}$ cells containing the HAC

The $21 \Delta \mathrm{qHAC}$, established in our previous study, is maintained in CHO cells (Katoh et al 2004), and possesses endogenous Hprt loci. To facilitate the detection of functional expression of the exogenously introduced 


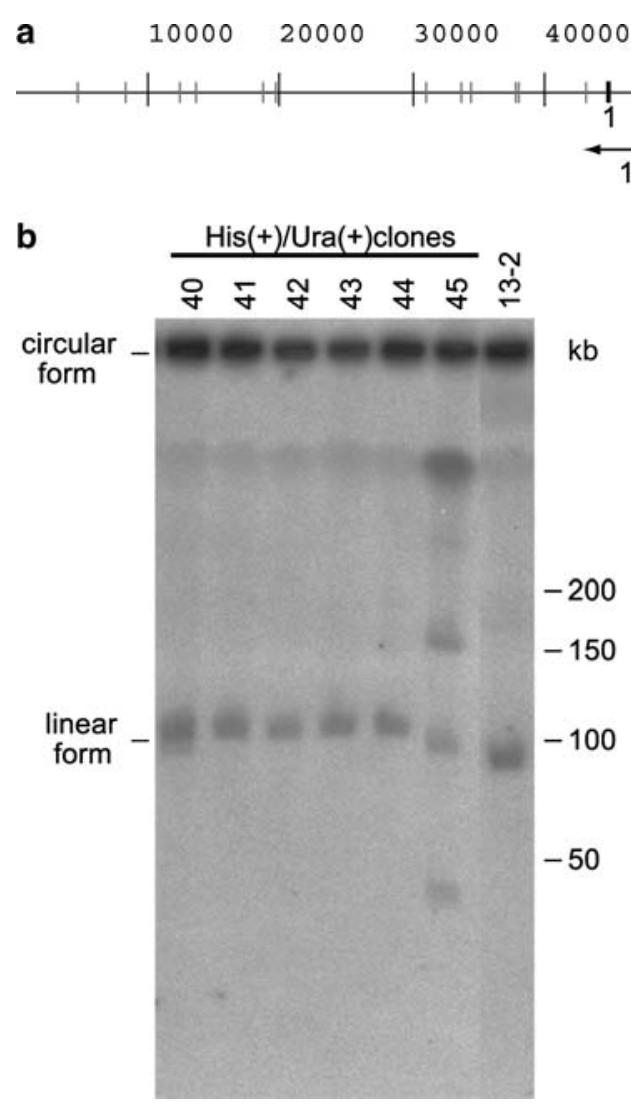

Fig. 2a-c Characterization of retrofitted HPRT-loxP/BAC/YACs by Southern blotting. a Restriction map of the $100 \mathrm{~kb} H P R T$-YAC isolated by TAR cloning. Nine exons disperse in four EcoRI fragments. b Chromosomal-size DNA was prepared in agarose

human HPRT allele, Hprt-deficient $\mathrm{CHO}$ derivatives were isolated. Null mutations in the Hprt gene were identified by the phenotype resistance to a purine analog 6-thioguanine (TG). Sixteen TG-resistant colonies were obtained out of $5 \times 10^{5}$ cells after drug selection; these were isolated and expanded for the following analysis. All of the clones were sensitive to HAT selection, suggesting that the endogenous Hprt loci were spontaneously disrupted. Two clones named $\Delta \mathrm{H} 1$ and $\Delta \mathrm{H} 2$ were arbitrarily selected and subjected to FISH analysis (Table 2). In both clones, it was observed that one copy of the independent HAC was not integrated into the host chromosomes in almost all of the metaphase spreads. This result indicated that the selection of Hprtdeficient derivatives did not affect the retention and integrity of the $\mathrm{HAC}$ vector.

Introduction of the HPRT-hCMV/loxP YAC/BAC into the $21 \mathrm{HAC}$ vector

The CHO $\mathrm{H} 8 \Delta \mathrm{H} 1$ cells were transfected with $H P R T$ hCMV/loxP YAC/BAC and the Cre expression vector pBS185, followed by G418 selection. The loxP site-spe-

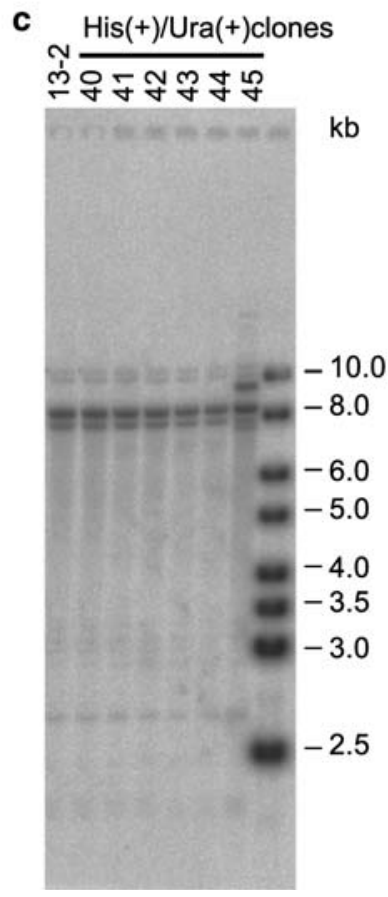

plugs from independent TAR YAC isolates containing the HPRT gene, exposed to X-rays, separated by pulsed field gel electrophoresis, and blot-hybridized with human Cot-1 DNA. The strong signals at the wells correspond to non-migrating circular molecules. The $100-\mathrm{kb}$ sized bands correspond to linearized molecules containing the gene and the TAR vector. c The EcoRI fragment

cific insertion of HPRT-BAC into the HAC vector was selected by reconstructing the functional Neo gene that confers G418 resistance (Fig. 1b). Thirty-two G418 resistant transfectants were obtained and propagated for the following analyses. PCR was performed in order to test the retention of the reconstructed Neo gene, intron 1 and exon 9 of the HPRT gene (Table 1). Although the presence of the Neo junction was detected in almost all of the clones, the deletion of the $5^{\prime}$ - and/or $3^{\prime}$-ends of the HPRT locus was observed in just a few clones. Retention of the functional HPRT locus was tested by culturing the cells in HAT containing medium. While 18 clones $(56 \%)$ retaining intron 1 and exon 9 were resistant to HAT selection, the remaining clones showing a deletion were HAT-sensitive (Table 1). Expression of the HPRT gene was tested by RT-PCR in the selected seven clones (Fig. 3). The transcripts were detected in the clones that retained the HPRT locus and exhibited a $\mathrm{HAT}^{\mathrm{R}}$ phenotype. The integrity of the HPRT-HAC was also assessed in the three representative clones by FISH analysis. An independent HAC was observed, without any aberration such as translocation, amplification, or integration into a host chromosome (Fig. 4). One copy of the HAC was detected in the majority of the meta- 
Table 1 PCR analysis and HAT selection of Neo resistant clones

\begin{tabular}{lllll}
\hline HPRT & & & $\begin{array}{l}\text { HAT } \\
\text { resistance }\end{array}$ & $\begin{array}{l}\text { Number of } N e o^{\mathrm{r}} \\
\text { transfectants }(\%)\end{array}$ \\
\cline { 1 - 4 } Neo junction & $5^{\prime}$-intron 1 & $3^{\prime}$-exon 9 & & \\
\hline+ & + & + & + & $18(56)$ \\
+ & + & + & - & $4(12)$ \\
+ & + & - & - & $3(9)$ \\
+ & - & + & - & $4(12)$ \\
- & - & - & - & $2(6)$ \\
- & + & + & + & $1(3)$ \\
\hline
\end{tabular}

phase spreads and interphase nuclei (Table 2), suggesting that the $\mathrm{HAC}$ is correctly maintained throughout mitotic division. These results indicate that the HPRT locus loaded into the HAC vector successfully achieved functional expression through transcription, utilizing its own promoter with the correct splicing of the transcript.

\section{Discussion}

The HAC vector described here has several advantages. It has the capacity to house large inserts, can be introduced into many types of mammalian cells, and is stably maintained as an independent chromosome in these cells (Kakeda et al 2005; Katoh et al 2004). It also provides a defined acceptor site for site-specific integration of a transgene, which assures reproducibility in the expression level of transgenes (Otsuki et al 2005). We used this HAC vector to introduce the native human HPRT locus into Hprt-deficient $\mathrm{CHO}$ cells, and the gene expression was demonstrated on a transcriptional level and by functional complementation of the HAT-sensitive phenotype. These results are the first demonstration that a genomic locus carried on the HAC vector can correct a genetic defect of cells in vitro through production of an adequate level of the required protein.

On the $21 \Delta$ pq HAC vector, the acceptor loxP site is surrounded by viral promoters that drive drug-resistant genes. The EGFP reporter gene driven by the CMV promoter was efficiently expressed from the $21 \Delta \mathrm{pq} H A C$ vector accommodated in an HT1080 hybrid (Katoh et al

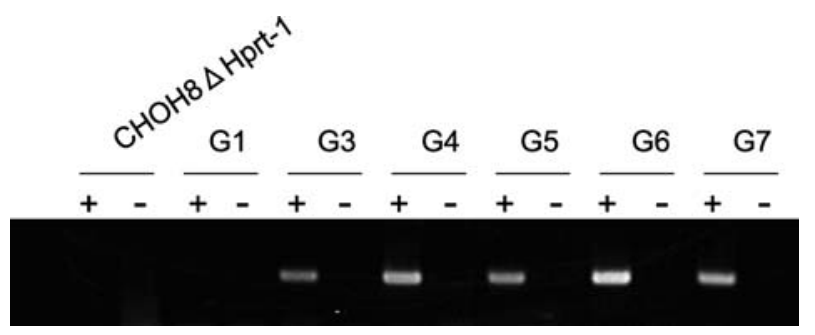

Fig. 3 RT-PCR analysis of $21 \Delta$ pqHAC vectors containing $H P R T$ loxP/BAC/YACs in Hprt-deficient CHO hybrid cells. RT-PCR was performed using a pair of primers specific to parts of exons 1-3 of the human HPRT gene. PCR products were detected in clones retaining the genomic locus that exhibited the $\mathrm{HAT}^{\mathrm{R}}$ phenotype (clones G3-G7). Plus and minus signs indicate the presence or absence of reverse transcriptase
2004). In contrast, the EGFP reporter gene driven by a minimal promoter with tissue specificity showed appropriate expression regulation in human mesenchymal stem cells during in vitro differentiation into osteoblasts only when the expression unit was surrounded by the insulators (Ren et al 2005). In this study, site-specific insertion of HPRT locus into the acceptor loxP site on the HAC vector showed functional expression of the HPRT gene. The HPRT-YAC/BAC used in this study has $\sim 40 \mathrm{~kb}$ upstream and $\sim 10 \mathrm{~kb}$ downstream regions around the substantial HPRT locus that include all regulatory elements needed for proper expression. Presence of these intervening sequences on both sides might ensure that the expression unit is insulated from being affected by the surrounding sequence at the acceptor $\operatorname{lox} P$ site.

Although the site-specific insertion of a genomic locus by $\mathrm{Cre} /$ loxP system has been reported by a few groups, the acceptor $\operatorname{lox} P$ sites were introduced in host chromosomes by random integration (Voet et al 2003). Call et al (2000) produced mouse ES cells carrying the acceptor $\operatorname{lox} P$ site and introduced a circularized linear YAC containing the $A M L 1$ and $K C N E 1$ locus from the human chromosome 21q22.3. Random integration of the acceptor $\operatorname{loxP}$ site led to the insertion of multiple copies of the loxP sites in the majority of transfectants. In a selected transfectant showing a single loxP site, correct insertion of a full-length intact YAC was detected in a relatively small fraction $(21 \%)$ of lox rec-

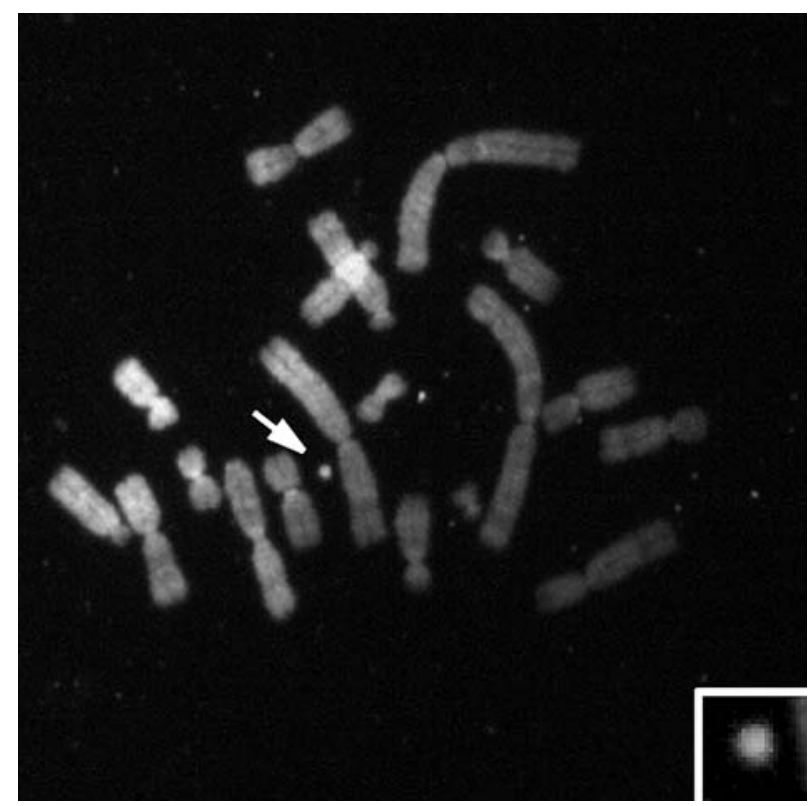

Fig. 4 FISH analysis of the $21 \Delta$ pqHAC vectors containing HPRTloxP/BAC/YACs in Hprt-deficient $\mathrm{CHO}$ hybrid cells. Shown are photomicrographs of representative metaphase spreads of hybrid cells containing a $\mathrm{HAC}$ vector in $\mathrm{CHO}$ background. The human chromosome fragment was detected by the human Cot-1 DNA probe as an independent copy without any chromosomal aberration such as amplification or translocation (arrow). Inset shows a clipping of the human chromosome fragment 
Table 2 FISH analysis of $21 \Delta$ pqHAC vectors in $\mathrm{CHO}$ cells

\begin{tabular}{|c|c|c|c|c|c|}
\hline \multirow[t]{2}{*}{ Clone } & \multirow[t]{2}{*}{$\begin{array}{l}\text { Number of analyzed } \\
\text { nuclei metaphase/interphase }\end{array}$} & \multicolumn{3}{|c|}{$\begin{array}{l}\text { Number of independent Cot } 1 \text { signals } \\
\text { per nucleus metaphase/interphase }\end{array}$} & \multirow[t]{2}{*}{$\begin{array}{l}\text { Retention rate of the } \\
\text { HAC in interphase }(\%)\end{array}$} \\
\hline & & 0 & 1 & 2 & \\
\hline$\Delta \mathrm{H} 1^{\mathrm{a}}$ & $50 / 100$ & $0 / 1$ & 48/96 & $1 / 3$ & 99 \\
\hline$\Delta \mathrm{H} 2^{\mathrm{a}}$ & $50 / 100$ & $1 / 5$ & $48 / 94$ & $0 / 1$ & 95 \\
\hline G1 & $50 / 100$ & $8 / 11$ & $40 / 85$ & $2 / 4$ & 89 \\
\hline G2 & $50 / 100$ & $5 / 12$ & $44 / 88$ & $1 / 0$ & 88 \\
\hline $\mathrm{G} 4^{\mathrm{a}}$ & $50 / 100$ & $6 / 5$ & $22 / 89$ & $0 / 6$ & 95 \\
\hline
\end{tabular}

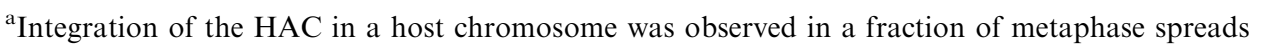

ombinants. This might be partly due to inefficiency in isolating intact circular YAC molecules by fractionation in gel electrophoresis (Compton et al 1999). In contrast to this report, the copy number of the acceptor loxP site is strictly regulated in our $\mathrm{HAC}$ vector, and the $\mathrm{HAC}$ carrying the gene of interest can be transferred to other cells such as mouse ES cells by microcell-mediated chromosome transfer (Shinohara et al 2001). In addition, conversion of the circular YAC to BAC by retrofitting facilitated subsequent isolation of the BAC and efficient loading of the genetic locus into the HAC vector in intact form. Thus, improvement in some aspects of site-specific insertion provides a more practical tool for functional analysis of genomic loci in the isogenic cell system.

In conclusion, combination of the HAC vector and TAR cloning system provides a practical system for the functional expression of a gene from the genomic locus. It might potentially offer a straightforward approach to testing the functional significance of a large number of gene-associated haplotypes by direct isolation of a genetic locus from individuals followed by transfer to an appropriate in vitro cell culture.

Acknowledgements We thank Drs. Yasuaki Shirayoshi, Akihiro Kurimasa, Hiroyuki Kugoh, Masaharu Hiratsuka, and Yasuhiro Kazuki (Tottori University) for valuable discussion, and Candice G.T. Tahimic for helpful suggestion. This study was supported in part by a Health and Labour Sciences Research Grant for Research on Human Genome, Tissue Engineering from the Ministry of Health, Labour and Welfare, Japan (MO, TI) and by the 21st Century COE Program from the Ministry of Education, Culture, Sports, Science and Technology of Japan (MO, TI): The Research Core for Chromosome Engineering Technology. FA was supported by Research Fellowships of the Japan Society for the Promotion of Science for Young Scientists.

\section{References}

Call LM, Moore CS, Stetten G, Gearhart JD (2000) A cre-lox recombination system for the targeted integration of circular yeast artificial chromosomes into embryonic stem cells. Hum Mol Genet 9:1745-1751

Caskey CT, Kruh GD (1979) The HPRT locus. Cell 16:1-9

Compton ST, Henning KA, Chen M, Mansoura MK, Ashlock MA (1999) An improved method for routine preparation of intact artificial chromosome DNA (340-1,000 kb) for transfection into human cells. Nucleic Acids Res 27:1762-1765
Conrad C, Vianna C, Freeman M, Davies P (2002) A polymorphic gene nested within an intron of the tau gene: implications for Alzheimer's disease. Proc Natl Acad Sci USA 99:7751-7756

Faustino NA, Cooper TA (2003) Pre-mRNA splicing and human disease. Genes Dev 17:419-437

Fukushige S, Sauer B (1992) Genomic targeting with a positiveselection lox integration vector allows highly reproducible gene expression in mammalian cells. Proc Natl Acad Sci USA 89:7905-7909

Garrick D, Fiering S, Martin DI, Whitelaw E (1998) Repeat-induced gene silencing in mammals. Nat Genet 18:56-59

Graveley BR (2001) Alternative splicing: increasing diversity in the proteomic world. Trends Genet 17:100-107

Hoehe MR, Timmermann B, Lehrach H (2003) Human interindividual DNA sequence variation in candidate genes, drug targets, the importance of haplotypes and pharmacogenomics. Curr Pharm Biotechnol 4:351-378

Ito H, Fukuda Y, Murata K, Kimura A (1983) Transformation of intact yeast cells treated with alkali cations. J Bacteriol 153:163-168

Kakeda M, Hiratsuka M, Nagata K, Kuroiwa Y, Kakitani M, Katoh M, Oshimura M, Tomizuka K (2005) Human artificial chromosome (HAC) vector provides long-term therapeutic transgene expression in normal human primary fibroblasts. Gene Ther 12:852-856

Katoh M, Ayabe F, Norikane S, Okada T, Masumoto H, Horike S, Shirayoshi Y, Oshimura M (2004) Construction of a novel human artificial chromosome vector for gene delivery. Biochem Biophys Res Commun 321:280-290

Kim JH, Leem SH, Sunwoo Y, Kouprina N (2003) Separation of long-range human TART gene haploypes by transformation-associated recombination cloning in yeast. Oncogene 22:2452-2456

Kouprina N, Larionov V (2003) Exploiting the yeast Saccharomyces cerevisiae for the study of the organization and evolution of complex genomes. FEMS Microbiol Rev 27:629-649

Kouprina N, Annab L, Graves J, Afshari C, Barrett JC, Resnick MA, Larionov V (1998) Functional copies of a human gene can be directly isolated by transformation-associated recombination cloning with a small $3^{\prime}$ end target sequence. Proc Natl Acad Sci USA 95:4469-4474

Kugoh H, Mitsuya K, Meguro M, Shigenami K, Schulz TC, Oshimura M (1999) Mouse A9 cells containing single human chromosomes for analysis of genomic imprinting. DNA Res 6:165-172

Landry JR, Mager DL, Wilhelm BT (2003) Complex controls: the role of alternative promoters in mammalian genomes. Trends Genet 19:640-648

Leem SH, Londono-Vallejo JA, Kim JH, Bui H, Tubacher E, Solomon G, Park JE, Horikawa I, Kouprina N, Barrett JC, Larionov V (2002) The human telomerase gene: complete genomic sequence and analysis of tandem repeat polymorphisms in intronic regions. Oncogene 21:769-777

Otsuki A, Tahimic CG, Tomimatsu N, Katoh M, Chen DJ, Kurimasa A, Oshimura M (2005) Construction of a novel expression system on a human artificial chromosome. Biochem Biophys Res Commun 329:1018-1025 
Ren X, Katoh M, Hoshiya H, Kurimasa A, Inoue T, Ayabe F, Shibata K, Toguchida J, Oshimura M (2005) A novel human artificial chromosome vector provides effective cell lineagespecific transgene expression in human mesenchymal stem cells. Stem Cells (in press; doi:10.1634/stemcells.2005-0021)

Shinohara T, Tomizuka K, Miyabara S, Takehara S, Kazuki Y, Inoue J, Katoh M, Nakane H, Ino A, Ohguma A, Ikegami S, Inokuchi K, Ishida I, Reeves RH, Oshimura M (2001) Mice containing a human chromosome 21 model behavioral impairment and cardiac anomalies of Down's syndrome. Hum Mol Genet 10:1163-1175

Tufarelli C, Stanley JA, Garrick D, Sharpe JA, Ayyub H, Wood WG, Higgs DR (2003) Transcription of antisense RNA leading to gene silencing and methylation as a novel cause of human genetic disease. Nat Genet 34:157-165

Voet T, Schoenmakers E, Carpentier S, Labaere C, Marynen P (2003) Controlled transgene dosage and PAC-mediated transgenesis in mice using a chromosomal vector. Genomics 82:596-605

Wen F, Li F, Xia H, Lu X, Zhang X, Li Y (2004) The impact of very short alternative splicing on protein structures and functions in the human genome. Trends Genet 20:232-236

Whitelaw E, Sutherland H, Kearns M, Morgan H, Weaving L, Garrick D (2001) Epigenetic effects on transgene expression. Methods Mol Biol 158:351-368 